\title{
A Neutrally Stratified Boundary-Layer A Comparison of Four Large-Eddy Simulation Computer Codes
}

\author{
A. ANDRÉN \\ Department of Meteorology, Uppsala University, Box 516, S-751 20 Uppsala, \\ Sweden \\ A. BROWN and P.J. MASON \\ Meteorological Office, London Road, Bracknell, Berkshire RG12 2SZ, \\ United Kingdom \\ J. GRAF and U. SCHUMANN \\ DLR, Institute of Atmospheric Physics, 82230 Oberpfaffenhofen, Germany \\ C.-H. MOENG \\ National Center for Atmospheric Research, Boulder, CO 80307, U.S.A. \\ F. T. M. NIEUWSTADT \\ Laboratory of Aero and Hydrodynamics, Delft University of Technology, Delft, \\ The Netherlands.
}

\begin{abstract}
A neutrally stratified Ekman layer limited in depth by a stress-free rigid lid has been simulated using four different large-eddy simulation (LES) computer codes. The resolution was chosen to be coarse so that the results might be sensitive to the details of the various SGS model formulations. Stochastic backscatter of subgrid kinetic energy and scalar variance was included in one SGS model.

Profiles of first and second moments, budgets of resolved-scale second moments, and spectra have been analyzed. It is found that significant differences in the lowest third of the boundary layer result from moderate changes in SGS eddy diffusivities or from the inclusion of backscatter.

To isolate effects of the various numerical methods, a set of tests with a fixed SGS K-profile were made. When run with a common SGS model the results from the four LES codes converge. This test indicates that the LES results are more sensitive to the SGS model formulations than to the various numerics.
\end{abstract}

\section{Nomenclature}

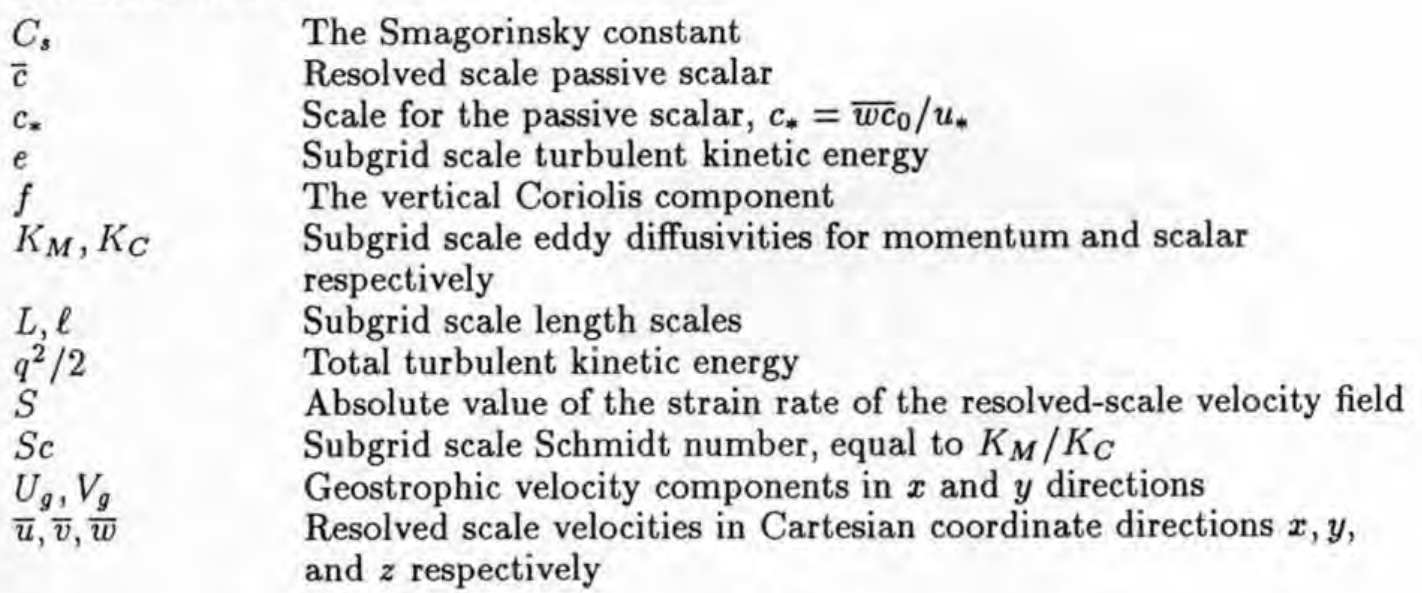




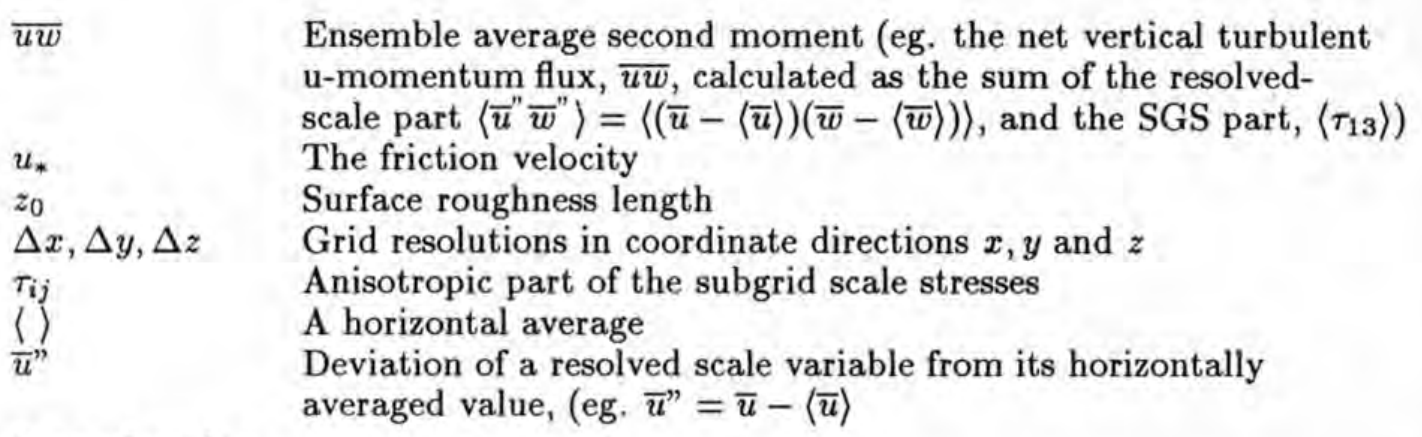

\section{Introduction}

For fine grids, LES should be insensitive to the particular choice of SGS parameterization, except close to the ground, where the turbulence scales must become smaller and an increased sensitivity to SGSparameterization is inevitable. However, results in the bulk of the boundary layer may be more sensitive to the subgrid model when using coarse grids which only marginally resolve the inertial subrange. In addition numerical details may also affect the simulation results.

In this paper, we present results from simulations of a particular flow by a set of LES codes that use a variety of SGS-parameterizations as well as different numerical methods. The flow studied is a neutrally stratified atmospheric boundary layer limited in depth by a stress-free rigid lid. A similar exercise was carried out by Nieuwstadt et. al. [1] (hereafter NMMS92) for a convective atmospheric boundary layer, using the same four LES codes as in the present study. NMMS92 found that all four LES simulations performed about equally well, i.e. differences, which typically were not greater than about $10 \%$, were found to lie within the scatter of available observations, even with a rather coarse resolution. It was therefore decided to extend the comparison to the (for LES) more challenging case, the neutral shear-driven boundary layer.

\section{Overview of the LES Codes}

We will refer to the four different LES computer codes as Mason's, Moeng's, Nieuwstadt's, and Schumann's. Detailed descriptions of the individual codes may be found in Mason [2]; Moeng [3]; Nieuwstadt and Brost [4] and Schmidt and Schumann [5], respectively. The backscatter model used in Mason's code is described in Mason and Thomson [6]. Here we will focus on the differences in the models' SGS-formulations and numerical techniques.

\section{Subgrid Scale Models}

Until quite recently, it was assumed that the only role of the SGS model 
in an LES is to dissipate the energy that is passed down to small scales through the energy cascade process. Typically this was achieved by using the Smagorinsky [7] formulation for the anisotropic part of the SGS stresses

$$
\tau_{i j}=-K_{M}\left(\frac{\partial \bar{u}_{i}}{\partial x_{j}}+\frac{\partial \bar{u}_{j}}{\partial x_{i}}\right),
$$

where the eddy viscosity, $K_{M}$, is given as $\ell^{2} S$. Mason's code, without the option of backscatter of kinetic energy and scalar variance, uses this formulation. In Moeng's, Nieuwstadt's and Schumann's codes, $K_{M}$ is given by

$$
K_{M}=C L e^{1 / 2},
$$

where the subgrid TKE is calculated from a prognostic equation. In Schumann's code, the $K_{M}$-formula follows from a simplified single-point closure scheme for the second moments of the subgrid fluctuations.

All the codes relate the SGS eddy diffusivity for scalars, $K_{C}$, to the SGS eddy diffusivity for momentum, $K_{M}$, through a constant SGS Schmidt number, $S c$.

To clarify differences in the SGS formulations we assume that shear production is balanced with dissipation in the SGS TKE budgets, and calculate $\ell=\left(K_{M} / S\right)^{1 / 2}$ for the three codes. These values can be compared with the value of $\ell$ used by Mason, which is prescribed directly without any explicit reference to grid resolution. Finally we can extract the values of $C_{s}$ using the common definition $\ell=C_{s}(\Delta x \Delta y \Delta z)^{1 / 3}$. Values of SGS Schmidt number, $\ell$ and $C_{s}$ used are given in Table 1 .

Table 1. Summary of interior values of $\ell$, SGS coefficients $\left(C_{s}\right)$, and SGS Schmidt numbers used in the individual LES codes.

\begin{tabular}{|l|ccc|}
\hline Code & $\ell(m)$ & $C_{s}$ & $S c$ \\
\hline Mason's & 10.0 & 0.17 & 0.70 \\
Moeng's & 13.6 & 0.24 & 0.33 \\
Nieuwstadt's & 12.7 & 0.22 & 0.33 \\
Schumann's & 7.6 & 0.13 & 0.42 \\
\hline
\end{tabular}

A longstanding problem in LES of neutrally stratified, high Reynolds number flows has been an inability to obtain a realistic logarithmic velocity profile in the surface layer, with excessive shear almost invariably found in the semi-resolved region close to the surface. The Smagorinsky model is purely dissipative and is deterministic as it assumes that subgrid motions are fully determined by the resolved 
motions. However, it has been recognized (e.g. Leslie and Quarini [8]) that SGS stresses should have stochastic fluctuations, and that the effect of these fluctuations is to 'backscatter' some energy from small to large scales, against the mean direction of energy transfer along the cascade. Mason and Thomson [6] used a stochastic subgrid forcing model in which the rates of backscatter of TKE and scalar variance were empirically tuned to give logarithmic mean profiles. In this study, Mason-Brown have performed simulations both with and without the backscatter effect.

\section{Numerical Solution Techniques}

Uniform vertical grid-structures are employed in all codes except Mason's. The various numerical schemes used are summarized below:

- Mason's and Nieuwstadt's codes: Advection terms are solved by a variance conserving second-order method, and time stepped by the leap-frog scheme with a weak time filter. Diffusion terms are second-order central differences and time advanced by the firstorder forward Euler method.

- Moeng's code: Advection terms conserve integrals of momentum and energy for the velocity fields, and volume integrated variances of the scalar fields. All horizontal derivatives are calculated by the pseudo-spectral method whereas vertical derivatives are approximated by second-order central differences. The second-order Adams-Bashforth time stepping is used for time integration.

- Schumann's code: Advection of velocity components is approximated by second-order energy-conserving central differences. Advection of scalars and subgrid energy is done using the secondorder upwind-scheme of Smolarkiewicz [9]. Second-order central differences are used for the diffusion terms. The second-order Adams-Bashforth scheme is used for time advancement.

All codes solve a Poisson equation for pressure through a mixed fastFourier and finite difference technique.

\section{Flow Set-up and Boundary Conditions}

We focus here on a planetary boundary-layer (PBL) at 45 degrees north, driven by a constant large-scale pressure gradient which, above the boundary layer, would balance a geostrophic wind of $\left(U_{g}, V_{g}\right)=$ $(10.0,0.0) m \cdot s^{-1}$. We include in these simulations both vertical and horizontal components of the Coriolis force. The flow was simulated with a grid of $40 \times 40 \times 40$ points in a horizontally periodic box of 
$4000 m \times 2000 m \times 1500 m$ in $x-, y-$ and $z$-directions, respectively. At the lower surface, we applied surface-layer similarity with a roughness length $z_{0}$ of $0.1 \mathrm{~m}$. A stress-free upper boundary was used. A passive scalar was introduced to the flow through a constant surface flux of $10^{-3} \mathrm{~kg} \cdot \mathrm{m}^{-2} \cdot \mathrm{s}^{-1}$.

All LES were initialized by the same steady state mean flow profile obtained from a PBL-model. To this mean flow, we added a small random perturbation in all velocity components. The simulations were run over a period of $0 \leq t \leq 10 / f s$, of which the subperiod $7 \leq t \leq 10 / f s$ were used to calculate statistics for analysis.

\section{Results}

A comparison of results from essentially the same flow as simulated here and observational data has been made by Mason and Brown [10]. Since the scatter in the data does not allow critical discrimination between the results, we limit ourselves to intercomparisons of the LES results from the different runs. A more extensive analysis may be found in Andrén et. al. [11].

\section{Time Series}

The resolved scales rapidly amplify during the first non-dimensional time unit, after which the turbulence intensity seems to have reached a quasi-steady state in all codes. The inertial oscillation in the mean fields in the different runs were found to be closely in phase. By averaging over the same time period we hope to have minimized any differences due to this oscillation. Whereas the mean velocity gradients vary only slowly in time the higher moments achieve steady state at the turbulence time scale and have been calculated with respect to horizontal averages. We expect them to be less sensitive to this oscillation.

Profiles

Normalized profiles of the first moments, $\langle\bar{u}\rangle,\langle\bar{v}\rangle$, and $\langle\bar{c}\rangle$, appear reasonably similar, within $\approx 10 \%$ of each other, and are not shown here.

However, it is revealing to look at the non-dimensional gradients in the surface layer, Fig.1. Except for the backscatter version of Mason's code these profiles all show excessive shear in the semi-resolved region.

Notable differences appear in the velocity variances, exemplified by $\overline{u^{2}} / u_{*}^{2}$ in Fig. 2. At a level of $z f / u *=0.03$ a $50 \%$ difference in total $\overline{u^{2}} / u_{*}^{2}$ is noted among the four results. The reduction in the magnitude of the peak in $\overline{u^{2}} / u_{*}^{2}$ when using backscatter in Mason's code was noted also by Mason and Thomson [6]. They concluded that the sharp peak in $\overline{u^{2}} / u_{*}^{2}$ in their non-backscatter runs was due 
to the excessive shear in the mean flow in the semi-resolved region.
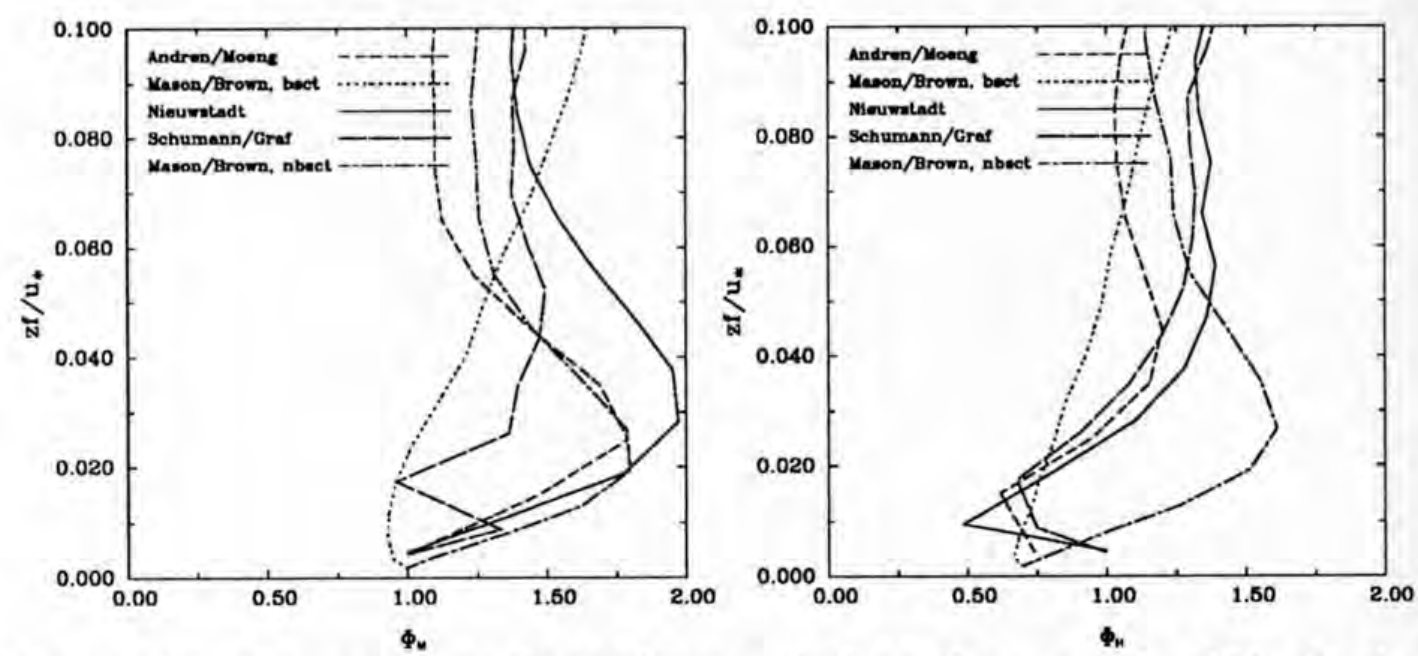

Fig. 1. Non-dimensional gradients of mean velocity and mean scalar fields. In the figures bsct/nbsct denotes a run with/without backscatter.
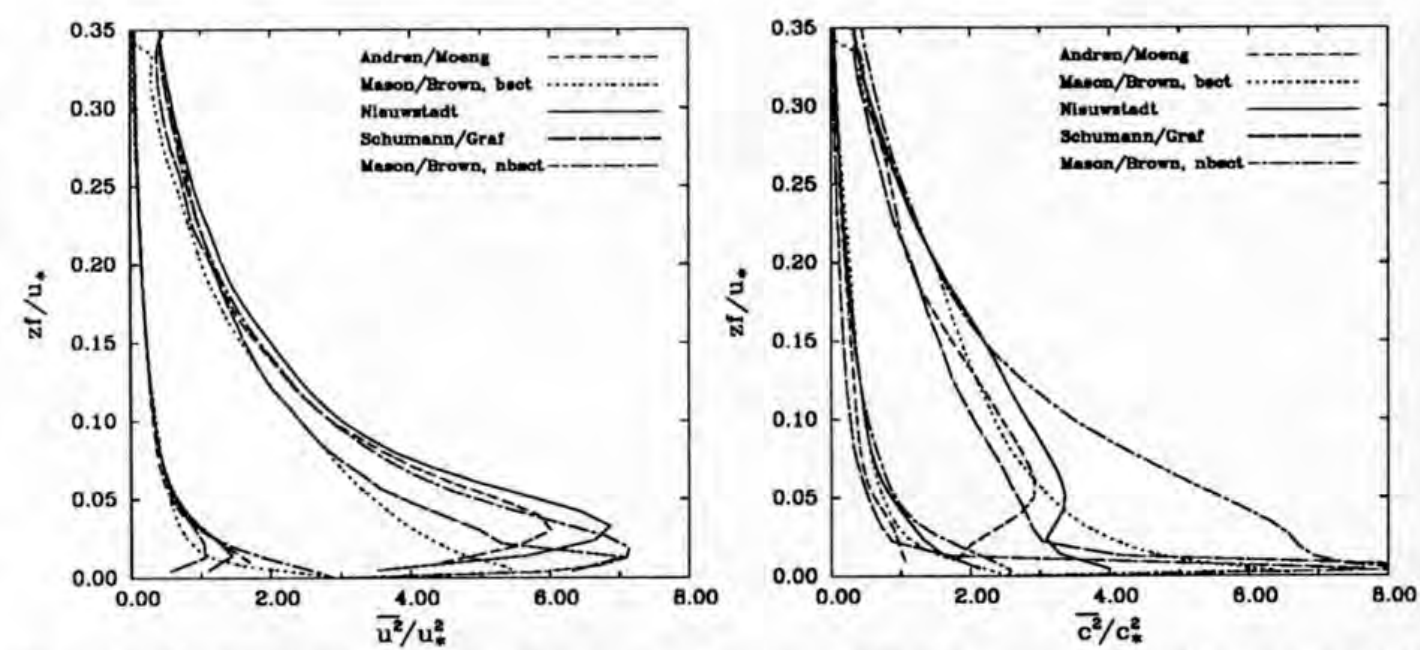

Fig. 2. Normalized velocity variances, showing both the sum of resolved and SGS contributions and the SGS part.

On the whole results from Schumann's code are closer to MasonBrown's backscatter runs. Since the SGS formulation used in Schumann's code for this non-buoyant case is equivalent to what is used in Moeng's and Nieuwstadt's codes, we must attribute the difference to the value of the Smagorinsky constant, $C_{s}$, and possibly to the differences in the numerical methods.

The SGS part of $\overline{c^{2}} / c_{*}^{2}$ is roughly the same for all models so that the differences in the total scalar variance observed in 
Fig. 2 must be due to the resolved motions. Mason-Brown's non-backscatter level of $\overline{c^{2}} / c_{*}^{2}$ is almost twice as large as the results from Moeng's, Nieuwstadt's and Schumann's codes. This is primarily due to the much larger Schmidt number used by Mason-Brown leading to smaller eddy diffusivities for the scalar, as confirmed by a short test run with reduced Schmidt number.
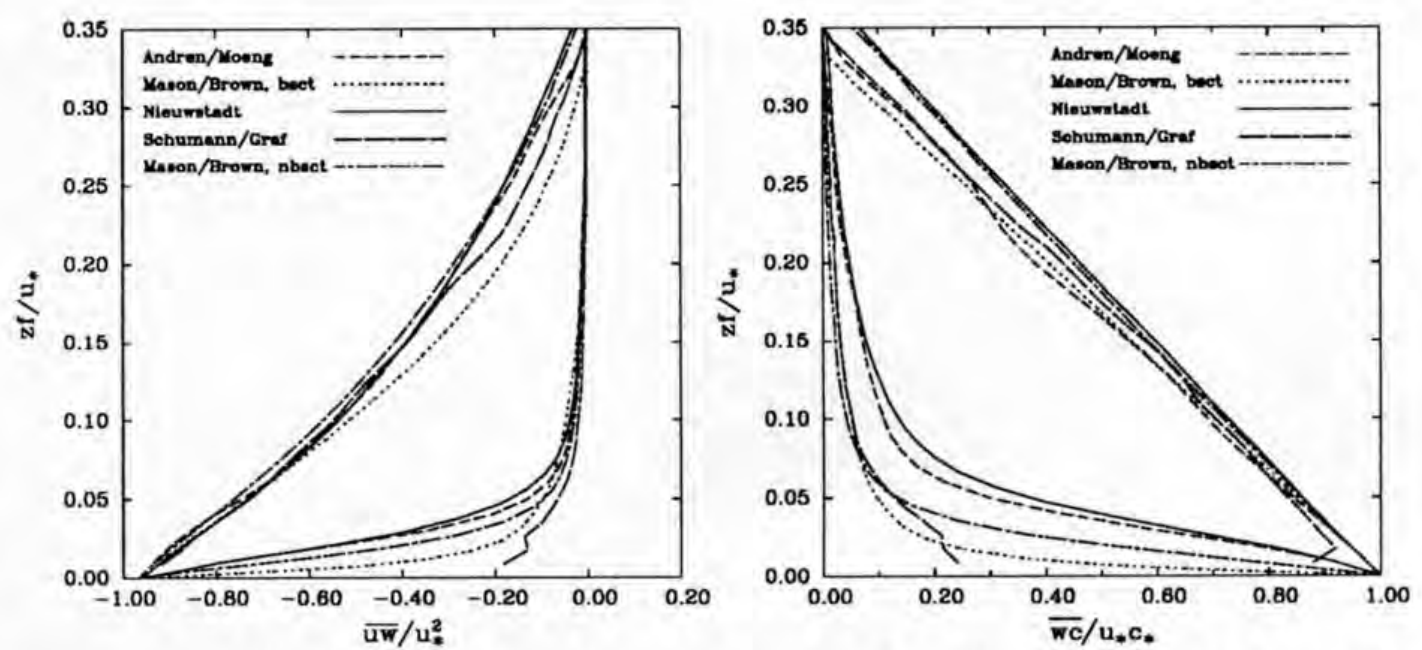

Fig. 3. Normalized vertical flux of $\mathrm{u}$-momentum and passive scalar $\mathrm{c}$, showing both the sum of resolved and SGS contributions and the SGS part.
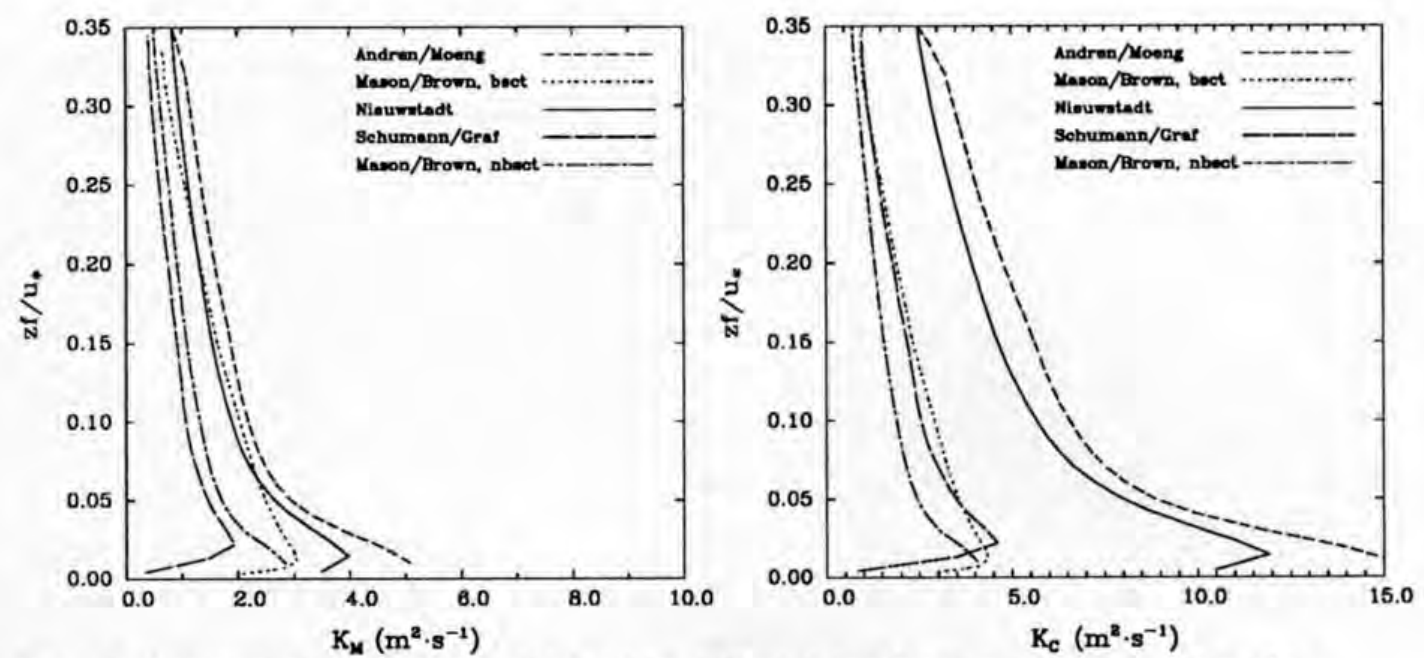

Fig. 4. Horizontally averaged SGS eddy diffusivities for momentum and scalar.

The large reduction in $\overline{c^{2}} / c_{*}^{2}$ when backscatter is included is again believed to be linked to the changes in near-surface gradients of the mean flow.

Profiles of total vertical fluxes of $\mathrm{u}$-momentum as well as total 
vertical fluxes of passive scalar $c$ agree well among the runs, Fig. 3. However, differences are found in the SGS part near the surface reflecting the differences in SGS eddy diffusivities and Schmidt numbers. Horizontally averaged SGS eddy diffusivities, Fig. 4, tend to form two groups with Mason-Brown's and Schumann-Graf's showing smaller values than Andrén-Moeng's and Nieuwstadt's.

Spectra

We have calculated energy spectra of the velocity and scalar fields and show here a sample of spectra at $z f / u_{*}=0.10$ in Fig. 5.
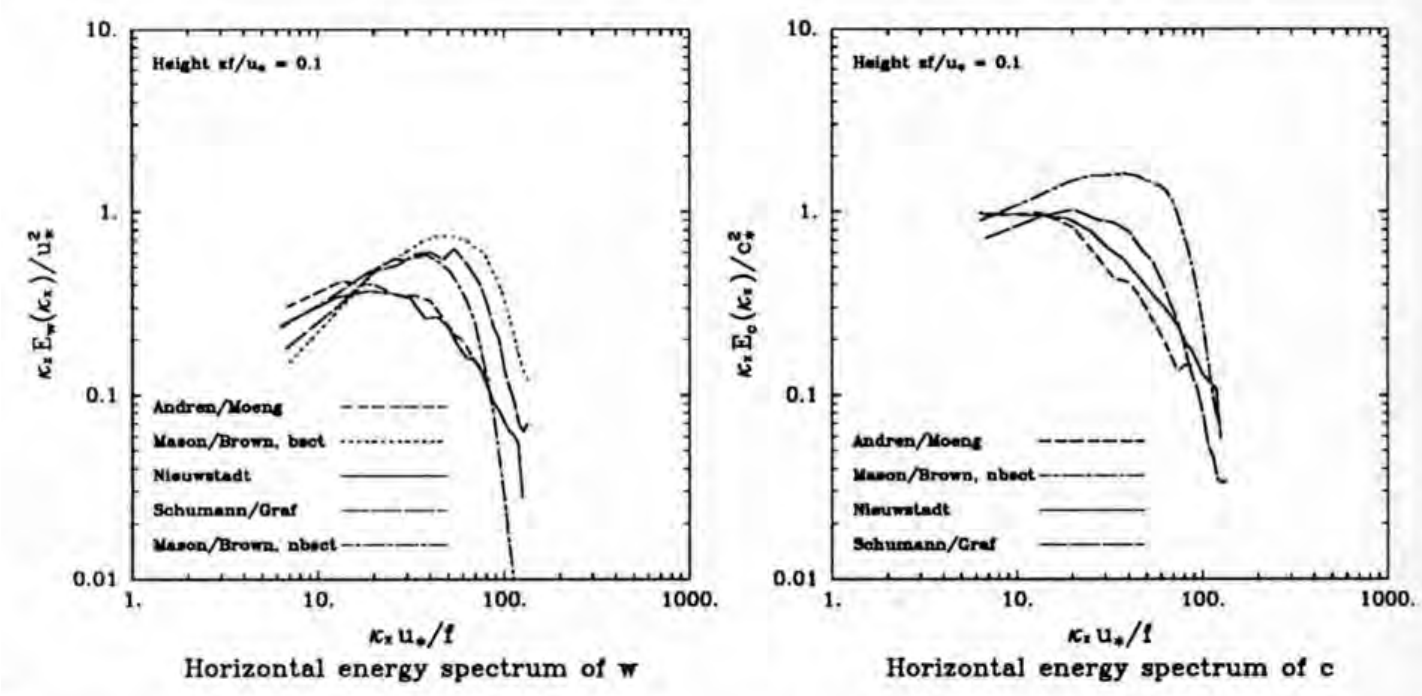

Fig. 5. Normalized spectra at a height of $z f / u_{*}=0.1$ of resolved-scale vertical velocity and passive scalar. Spectral estimates have been multiplied by a factor 1000 in order to provide more reasonable labels along the ordinate.

These spectra show that smaller SGS eddy diffusivities push spectral peaks towards higher wavenumbers. Results from Moeng's and Nieuwstadt's codes have spectral peaks at nondimensional wavenumbers around 20. Spectra from Mason's and Schumann's codes peak at nondimensional wavenumbers around $30-40$, and show a steeper fall off at high wavenumbers.

With backscatter in Mason's code the smallest resolved scales experience a continuous random excitation, shifting spectral peaks to higher wavenumbers. A similar effect is obtained with reduced values of SGS eddy diffusivities. By reducing SGS $K_{M}$ and $K_{C}$, one effectively reduces the energy dissipation out of the high wavenumbers of resolved fields. With the same energy cascade from larger eddies, this results in more energy in the higher wavenumbers of the resolved fields. 


\section{Discussion}

The differences among the four LES codes can only be due to the differences in their SGS eddy diffusivities and their numerics. To isolate possible numerical effects we ran all codes with fixed SGS diffusivities which were set equal to the average $K$-profiles from MasonBrown's non-backscatter run with uniform grid, shown in Fig. 4. (We also allowed for no backscatter in Mason's SGS model). A short test period was used instead of rerunning the whole simulation. Starting from the individual results at $t=7 / f$ and integrating to $t=9 / f$, the last non-dimensional time unit was used for averaging.
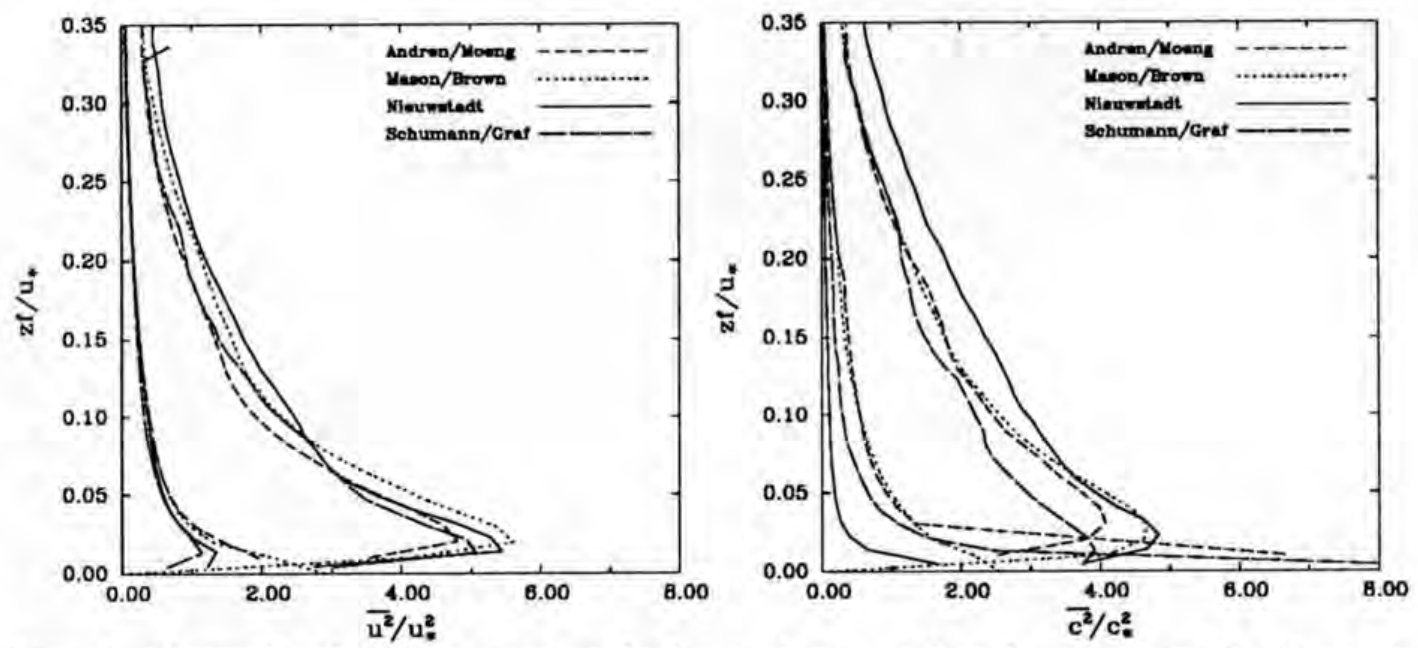

Fig. 6. Normalized $\mathrm{x}$-component velocity variance and scalar variance for the test run with fixed profiles of SGS eddy diffusivities. The run by Mason-Brown is on a uniform vertical grid, without backscatter.

Fig. 6 shows that the variances $\overline{u^{2}} / u_{*}^{2}$ and $\overline{c^{2}} / c_{*}^{2}$ now agree very well among the different codes. The Smolarkiewicz scheme which produces some numerical diffusion may be responsible for slightly smaller scalar variances in Schumann's code.

The spectra are particularly interesting since they directly give us the response of different scales to differences in the numerics. As described above, the numerical schemes used by Mason-Brown and Nieuwstadt are identical, and accordingly they should produce similar results (within the limits given by the rather short sampling period of the test). This is the case in the lower part of the boundary layer, as shown in Fig. 7. However, in the upper half of the boundary layer, we still find a steeper decrease in the runs by Mason-Brown and SchumannGraf than in the runs by Andrén-Moeng and Nieuwstadt (not shown). For the latter two codes, the scalar exhibits an almost flat spectrum. 

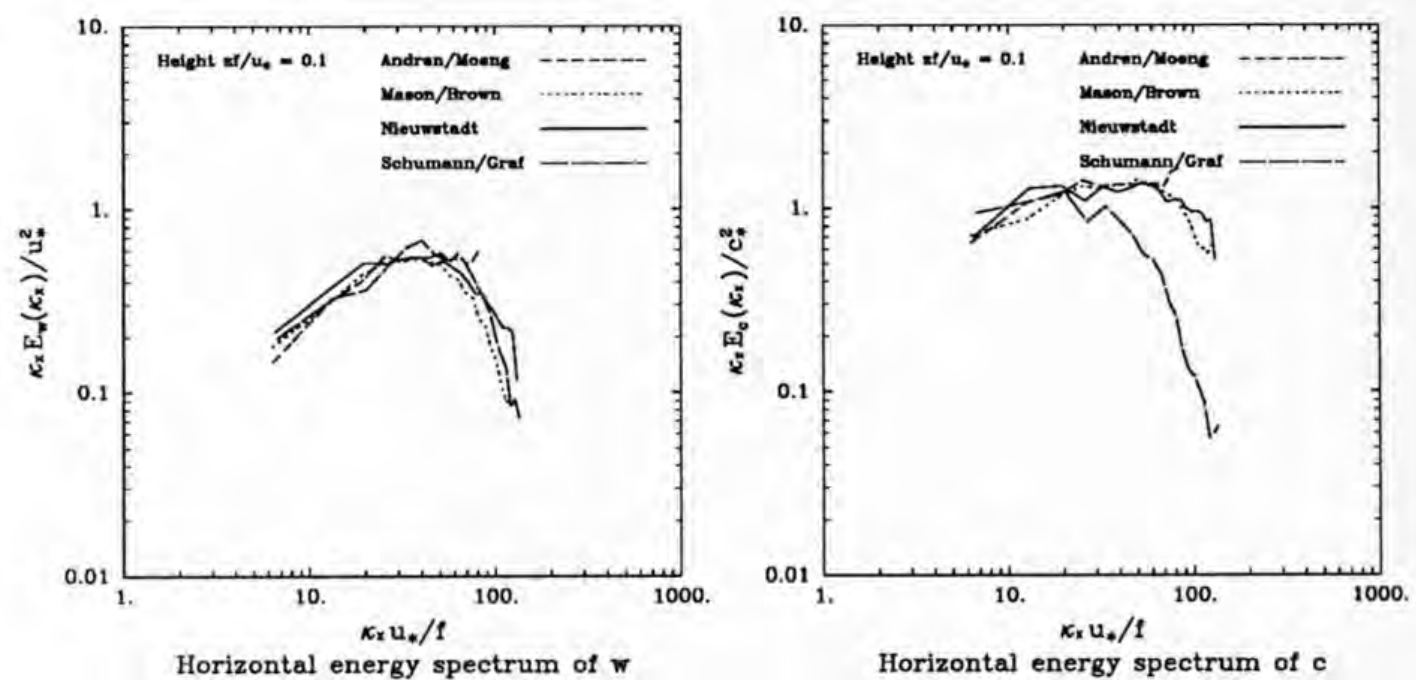

Fig. 7. Spectra as in Fig. 5 but for the test run with fixed profiles of SGS eddy diffusivities. The run by Mason-Brown is on a uniform vertical grid, without backscatter.

We notice that Mason-Brown's spectra show slightly more energy at higher wavenumbers than their original non-backscatter run. Schumann-Graf's spectra show a similar but smaller change. Their results for the passive scalar also indicate the dispersive property of the Smolarkiewicz advection scheme.

The convergence of spectral shapes at lower levels with differences still remaining at higher levels, where turbulence timescales are larger, may be an indication that the test run is too short for all spectra to reach stationary conditions. Thus we do not view the remaining differences between the codes as indications of effects of different numerical solution schemes. Rather we take the general convergency of profiles, budgets (not shown), and, to a lesser extent, spectra as an indication that the numerics have very little impact on the LES solutions, compared with the SGS effect.

\section{Conclusions}

A shallow neutrally stratified PBL has been simulated using four different LES codes. We have found that second moments as well as the budgets of second moments (not shown) are sensitive to the magnitude of the SGS eddy diffusivities. In general we found that statistics of this neutrally stratified flow were more sensitive to the SGS model than corresponding statistics in the convective flow studied by NMMS92.

With smaller SGS eddy diffusivities we find generally more intense 
resolved scale motions in the lower part of the boundary layer, and that spectra peak at higher wavenumbers. The inclusion of stochastic backscatter has a similar effect on the spectra and on the resolvedscale second moments and budgets. Differences between backscatter and non-backscatter runs are in general largest close to the surface, but also noticeable in the lower one-third of the boundary layer.

For the mean fields, our findings support the result of Mason and Thomson [6] that the failure of the Smagorinsky SGS model in producing logarithmic mean gradients near the wall cannot be corrected by changing the value of the Smagorinsky coefficient.

A short test run with fixed profiles of SGS eddy diffusivities showed that the effect of the differences in numerical schemes is minor.

\section{References}

[1] Nieuwstadt, F.T.M., Mason, P.J., Moeng, C.-H. and Schumann, U., Large-eddy simulation of the convective boundary layer: A comparison of four computer codes. In Turbulent Shear Flows 8 (1992) Eds. F. Durst et. al., Springer-Verlag, Berlin, 343-367.

[2] Mason, P.J., Large-eddy simulation of the convective boundary layer. J. Atmos. Sci. 46 (1989) 1492-1516.

[3] Moeng, C.-H., A large-eddy-simulation model for the study of planetary boundarylayer turbulence. J. Atmos. Sci. 41 (1984) 2052-2062.

[4] Nieuwstadt, F.T.M. and Brost, R.A., The decay of convective turbulence. J. Atmos. Sci. 43 (1986) 532-546.

[5] Schmidt, H. and Schumann, U., Coherent structure of the convective boundary layer derived from large-eddy simulation. J. Fluid Mech. 200 (1989) 511-562.

[6] Mason, P.J. and Thomson, D.J., Stochastic backscatter in large-eddy simulation of boundary layers. J. Fluid Mech. 242 (1992) 51-78.

[7] Smagorinsky, J., General circulation experiments with the primitive equations. Part 1: The basic experiment. Mon. Wea. Rev. 91 (1963) 99-164.

[8] Leslie, D.C., and Quarini, G.L., The application of turbulence theory to the formulation of subgrid modelling procedures. J. Fluid Mech, 91 (1979) 65-91.

[9] Smolarkiewicz, P.K., A fully multidimensional positive definite advection transport algorithm with small implicit diffusion. J. Comput. Phys. 54 (1984) 325-362.

[10] Mason, P.J. and Brown, A.R., The sensitivity of large-eddy simulations of turbulent shear flow to subgrid models. Submitted to Boundary-Layer Meteor. (1993)

[11] Andrén, A., Brown, A., Graf, J., Moeng, C.-H., Mason, P.J., Nieuwstadt, F.T.M. and Schumann, U., Large-eddy simulation of a neutrally stratified boundary layer: A comparison of four computer codes. Submitted to Quart. J. Roy. Meteor. Soc. (1993). 